\title{
Warm-up or Proprioceptive Neuromuscular Facilitation are Unnecessary before Anaerobic or Aerobic Power Exercise?
}

\section{Carlos Alexandre Fett ${ }^{1^{*}}$ (D) Karla Regia Ferreira Viana Figueiredo ${ }^{1}$ (D) Almir de Franca Ferraz ${ }^{1,2,3}$ (iD, Fabiula Lebbeck ${ }^{1}$ (D) Camila Fernanda ${ }^{4}$ (D) and Waléria Christiane Rezende Fett ${ }^{1}$ (D)}

${ }^{1}$ Federal University of Mato Grosso, Brazil

${ }^{2}$ São Judas Tadeu University, Brazil

${ }^{3}$ Mato Grosso Military Police Teaching Directorate, Brazil

${ }^{4}$ University of São Paulo, Brazil

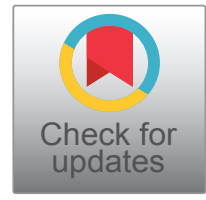

*Corresponding author: Carlos Alexandre Fett, Federal University of Mato Grosso, Av. Fernando Correa da Costa, s/n, University City, Sports Gym, 78060-900 - Cuiabá - MT, Brazil

\begin{abstract}
Preparatory activities for physical exercise and/or sports competition are carried out with the improving performance and preventing injuries. Traditional Warm-up (TW), such as jogging and Proprioceptive Neuromuscular Facilitation (PNF) stretching, are two examples of activities used for this purpose. However, there is no consensus in the literature about their efficacy, and which of them is the best. The aim of this study was to compare the effects of TW and PNF on anaerobic power (AnP) and Aerobic Power (AP) and on physiological variables. In this study 10 (ten) men aged between 18 and 30 years, physically active, non-athletes, were evaluated in an external environment with an average temperature of $34^{\circ} \mathrm{C}$, in a crossover system in 04 different time intervals: TW (M1), PNF (M2), TW (M3) and PNF (M4) before the vertical jump test and $20 \mathrm{~m}$ Yo-Yo tests, with an interval of one week between them. The AnP and AP values were calculated; the post-test lactate and heart rate were measured, and the volunteers were asked about Delayed Onset Muscle Soreness (DOMS) 24h the tests. One-way ANOVA was carried out to test the 04 time intervals, with significance established at $5 \%$. There were no differences between the variables in the different time intervals. There was no difference in physical performance in any of the types of pre-exercise preparation activities. Furthermore, TW with elevation of body temperature and PNF that improve muscle length appeared to be dispensable for active young men in a warm environment.
\end{abstract}

\section{Keywords}

Warm-up, Anaerobic Power, Aerobic Power

\section{Introduction}

Physical exercise demands greater production of work per unit of time from the body, thereby overloading all the organic systems to a greater or lesser time interval; and causes global functional and structural changes to express what is happening at cellular level. These participations are defined by the intensity and duration of exercise, which normally has an inverse proportion between them [1]. Sports and physical activities normally have previous, acute or chronic preparation. Acute preparation stimulates the body for the more intense main activity, and is normally composed of general or specific, active or passive warming-up or stretching [2]. Chronic preparation consists of sequential planning of the physical exercise programs, with specificities determined by the purpose of training [1]. The purpose of the present study was to discuss the first situation.

Warming-up is performed with a view to increasing body temperature, diminishing muscle and blood viscosity, increasing neuromuscular transmissions, activating the peripheral proprioceptors, signaling the CNS to increase the metabolic production of energy [2,3]. Active warming-up mainly modulates metabolic changes, while the passive type increases the temperature of the body nucleus without spending important energy. Studies have demonstrated that warming-up in hot water 
at $40{ }^{\circ} \mathrm{C}$ was as beneficial as intermittent high intensity physical activity [4], while an elevated environment temperature reduced the performance of football players [5].

Warming-up also seeks to improve performance and reduce lesions in physical exercises $[6,7]$. In those of short duration and high intensity, it potentiates the rapid production of energy, producing more intense contractions; and in those of long duration, previously activates the energy production of the aerobic component; reduces the slow aerobic component and toxic metabolites, such as lactic acid. However, is not defined as regards the combination of intensity, duration and interval for each type of exercise [4] and the environmental interferences combined with physiological factors stimulated by physical exercise. There is little evidence of improvement in sports performance, commonly verified in studies with few participants, focused on physiological changes and not on physical performance, therefore studies are necessary to demonstrate these different conditions [2].

Normally, stretching is performed as a means of warming-up, but for this an increase in body temperature is necessary; however, stretching does not meet the requirements of this condition. Stretching is performed with a view to maintaining or increasing articular mobility, and may indirectly potentiate physical performance by the increase in articular amplitude and elastic potential of the musculature, favoring activities that demand greater muscle strength and power, reducing injuries, especially in eccentric contractions $[8,9]$.

Stretching exercises are divided into the following categories: passive, ballistic or active stretching; forced and Proprioceptive Neuromuscular Facilitation (PNF). Studies have demonstrated that passive and ballistic stretching diminish the muscle capacity for generating tension and to exert force against resistance $[8,10]$; the ballistic type was superior to these in activities involving balance and agility [11] or made no difference in the production of force and prevention ofinjuries; [12] PNF increased articular amplitude and kicking velocity of young football players [13], but little has been investigated about aerobic performance. Moreover, PNF stretching showed no difference in maximum voluntary contraction in electromyography of the vastus lateralis and rectus femoris [14], and worsened the maximum isometric when compared with the static strength, [15] however, it has not been studied with regard to being performed before anaerobic power exercises (AnP) and Aerobic Power (AP) exercises.

The effects of stretching on preventing lesions and performance are contradictory in the literature, mainly due to the possible variations among techniques and sports involved $[7,16,17]$. In a systematic review, Hebert, et al. [18] concluded that stretching before, after; or before and after physical training did not produce clinically important reduction in Delayed Onset Muscle Soreness (DOMS). The authors of the present study obtained a similar result in their group when young women were induced to maximum eccentric biceps curl, divided into groups massage, passive stretching, PNF stretching, or rest (control), in which at the time intervals of 24, 48, 72, 96 and 120 hours post-test, the greatest reduction obtained was shown for massage and the worst result was obtained with passive stretching, and without difference between PNF and rest [19]. It has also been pointed out that stretching performed with a view to increasing the amplitude of movement worsened strength and power performance, without interference for ballistic stretching, but increased the amplitude of movement and are beneficial in the long term in sports that require this condition [17].

In this regard, the present study investigates the effects of traditional warming-up (TW) x PNF performed before an activity of short duration for AnP and of medium duration for AP.

\section{Methods}

\section{Participants}

The project was approved by the Research Ethics Committee of University Hospital Júlio Müller, Protocol $n^{\circ}$. 658/CEP/HUJM/09 following The Helsinki Statement on Health in All Policies. All the volunteers signed the term of free and informed consent to participating in the research study. Therefore, the authors declare that there was no conflict of interests.

Inclusion criterion was that the volunteers had to be active, defined by physical activities practiced two to three times per week, in addition to participating in sports classes of the Physical Education Faculty, but not being athletes. The students of the Physical Education Faculty were invited to participating of the present study by direct contact and posters. For the study, 180 male students were contacted, and those excluded were under 18 or older than 30 years of age; who had any type of bone or muscle injury that was impair the participation in the tests, and athletes. Thus 10 (ten) remained, who fulfilled the requisites and responded to the call of the study. The authors conducted this study in Cuiabá, Mato Grosso, Brazil, one of the hottest regions in the country. The mean annual temperature is $27^{\circ} \mathrm{C}$ and between October and March, a temperature of $40^{\circ} \mathrm{C}$ is frequently attained. The mean in the month of October, when the tests were performed was $34^{\circ} \mathrm{C}[20]$.

\section{Study design}

The volunteers performed the treatments in four moments (M): TW (M1), PNF (M2), TW (M3) and PNF (M4) followed by the vertical jump test (VJ), measuring anaerobic (AnP) and the $20 \mathrm{~m}$ Yo-Yo test, to obtain aerobic power (AP); collection of Lactic acid (Lac) and heart rate (HR) data, with an interval of one week between 


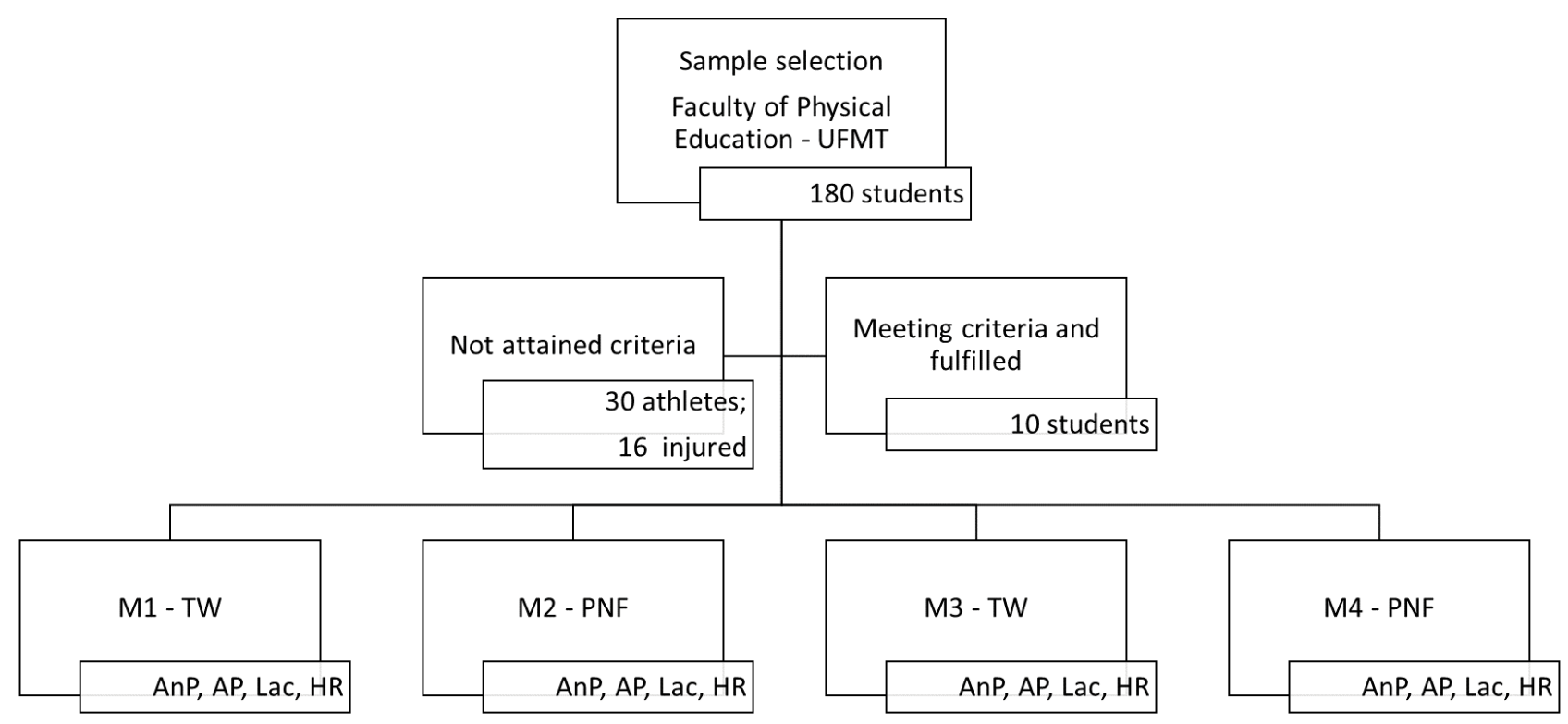

Figure 1: Experimental design.

The authors invited 180 male students; 46 did not meet the inclusion criteria, and only 10 who did meet these conditions volunteered. There was a one-week interval between each moment of tests performance: (M1, M2, M3 and M4) With traditional warming-up, that consist in a light jogging (Borg de 3-5) or Proprioceptive Neuromuscular Facilitation (PNF) stretching, each with a duration of $7 \mathrm{~min}$, before the Anaerobic Power (AnP) followed by the Aerobic Power (AP) tests, and after that capillary lactate (Lac) and Heart Rate (HR) data collection.

the tests (Figure 1). In the twenty-four hours preceding the tests, the volunteers had to avoid vigorous physical activities, use of alcoholic beverages or exaggerated caffeine consumption.

\section{Anthropometric measurements}

The authors evaluated body weight in kilograms and height in meters (scale: Soehnle Professional (Germany)), with precision of $0.1 \mathrm{~kg}$ and $0.5 \mathrm{~cm}$ respectively, volunteers dressed in shorts only [21].

\section{Traditional Warming-UP (TW)}

Were performed 07 mins., of jogging with intensity between light and moderate ( 3 to 5 ) on the modified Borg scale of $0-10$.

\section{Proprioceptive Neuromuscular Facilitation Stretch- ing (PNF)}

The target muscle group was stretched by moving the joint/articulation up to is maximum amplitude of movement for 10 seconds; being pre-elongated, it was isometrically contracted against the resistance of a helper for 10 seconds maintaining the same position, and lastly the muscle was relaxed, performing another stretch in a static manner up to the greatest point of limitation for another 10 seconds. The isometric contraction intensity was approximately $60 \%$ of the maximum, being in agreement with contemporary recommendations [22]. PNF stretching was performed for $07 \mathrm{~min}$., with 02 series of 30 seconds for each muscle group, in which:

- Chest: Performed with the volunteer standing up, shoulders in horizontal extension at approximate- ly $90^{\circ}$, palms of the hands facing outwards.

- Back: With the volunteer in the orthostatic position, feet slightly apart, knees semi-flexed and arms crossed over the chest, performing scapular abduction. The helper supported the volunteer's back with his own weight, holding onto the elbows, performing stretching.

- Lumbar and hamstrings: The volunteer seated on a mat, legs extended, performing flexion of the hip and trunk over the legs, trying to reach his limit of flexibility in the direction of the feet.

- Quadriceps: Volunteer was in ventral lying on a mat. With the aid of a helper supported on the front part of the ankles, inducing knee flexion so that the foot, in plantar flexion would approximate the gluteal muscles.

- Hamstrings and gluteus: The volunteer was lying with the back completed supported on the mat, legs extended on the ground, performing unilateral flexion of the hip, with the aid of the helper, one of the leg, without flexing the knee, until the articular limit was reached. The helper held the heel, applying a force, in order to approximate the lower member to the thorax. The other leg remained extended on the ground, where the helper supported the foot on the volunteer's thigh, avoiding flexure of the hip and knee.

- Calf: In the same position as that of the previous exercise, with one leg extended until the articular angle of the hip was equal to $90^{\circ}$, inducing dorsiflexion of the foot. 
- Hip abductors: With the volunteer lying down, back on the mat, performing a $90^{\circ}$ flexion of the hip, elevating the lower limbs. With help, being held by the internal and distal surface of the thigh, the volunteer performed abduction of the hip.

\section{Anaerobic Power Test (ANP)}

The authors used the VJ, indirectly measuring the muscle power of the lower limbs [23]. The test was performed with the individual placed beside a wall where he performed the high jump starting from the static position, being allowed to move his arms, and the highest jump obtained in three attempts was measured. The power of the lower limbs was calculated by means of the following equation:

$$
P k g m . s=2.21 \times k g \times \sqrt{D}
$$

\section{Where:}

Pkgm.s = power in kg per meters per seconds;

$\mathrm{kg}=$ body mass

$\mathrm{D}=$ difference between marks in meters.

\section{Aerobic Power Test (AE)}

The $20 \mathrm{~m}$ Yo-Yo run test was used, composed of 21 stages, starting with a speed of $8.5 \mathrm{~km} / \mathrm{h}$, with $0.5 \mathrm{~km} / \mathrm{h}$ per stage being added, with the frequency being recording followed audio bleep clues played from a CD player. Each stage lasted approximately 1 minute, performing between 7 and 15 back and forth runs, with adjustment of velocity after every 2 or 3 runs. The space was demarcated by two lines on the ground, where the volunteer was warned to increase his rhythm if he did not attain this distance, and the test was stopped if the voluntary was not able to following the rhythm. To predict the $\mathrm{VO}_{2}$ max, the following equation was used:

$$
Y=-24,4+6,0 X
$$

\section{Where:}

$\mathrm{Y}=\mathrm{VO}_{2}$ max predicted $\left(\mathrm{ml}^{\mathrm{kg}} \mathrm{kg}^{-1} \cdot \mathrm{min}^{-1}\right) ;$

$X=$ Velocity attained $(\mathrm{km} / \mathrm{h})$ in the final stage of the test.

\section{Lactate and heart rate data}

Lactate and HR data were measured immediately after the AP test. Lactate was measured with a portable apparatus (Accutrend ${ }^{\circledR}$ Lactate; Accusport Bm-Lactate; Manufacturer: Roche 2007), after piercing (Accucheck Softclix) the forefinger, and using reagent tapes of the same brand. HR was measured with a portable frequency meter of the $\operatorname{POLAR}^{\circledR}$ brand.

\section{Delayed Onset Muscle Soreness (DOMS)}

The numerical pain scale was used $24 \mathrm{~h}$ after the tests.
Table 1: Age, bodymass, statureandbodymass index (BMI) of volunteers.

\begin{tabular}{|l|l|}
\hline Variables & Mean \pm SD \\
\hline Age (years) & $23.2 \pm 2.85$ \\
\hline Bodymass $(\mathbf{k g})$ & $70.7 \pm 2.27$ \\
\hline Stature $(\mathbf{m})$ & $1.73 \pm 0.04$ \\
\hline BMI $\left(\mathbf{k g} / \mathbf{m}^{2}\right)$ & $23.44 \pm 7.27$ \\
\hline
\end{tabular}

\section{Statistical analysis}

One-way ANOVA was used to compare the group in the 04 moments, followed by the Tukey-Kramer posttest for paired and parametric measurements. Significant difference was considered $\mathrm{P}<0.05$ with an interval of confidence of $95 \%$. The results were reported as mean \pm SD.

\section{Results}

There was no report of joint/articular injurie or use of medications that would affect HR and energy metabolism. The sample was homogeneous, composed of eutrophic young men (Table 1).

There was no significant increase in DOMS evaluated in the hours subsequent to the tests and in 24 hours. No significant difference was observed among the variables measured in the four time intervals (Figure 2).

\section{Discussion}

The main finding of this study was that there were no difference in performance of an AnP and AP activity, and physiological variables with regard to performing TW or PNF. Differently from the above-mentioned observation, there are demonstrations in which warming-up with elevation in body temperature favored performance $[4,24]$. Pre-exercise stretching exercises have presented controversies, such as no interference of stretching in performance [12]; effect of worsening on strength and power exercises $[8,10]$ and improvement of performance in tests $[9,12]$. Different protocols, types of stretching, publics investigated, level of performance and sex may justify these differences. Following we will discuss the differences in these conditionings.

\section{Warming-up}

Warming-up exercises might potentiate muscle efficiency for energy production and intense muscle contraction with possible reduction in metabolites such as lactate [4]. Thus in the present study, we investigate the changes in these variables by comparing TW and PNF, however, none of them changed significantly. Apparently, warming-up the body, a concept defended in sports, in physical education and in the gyms, seemed to have no influence on performance in the present study, because PNF did not elevate the body temperature, and did not differ from the performance obtained by TW. However, differently from that which we observed, in 


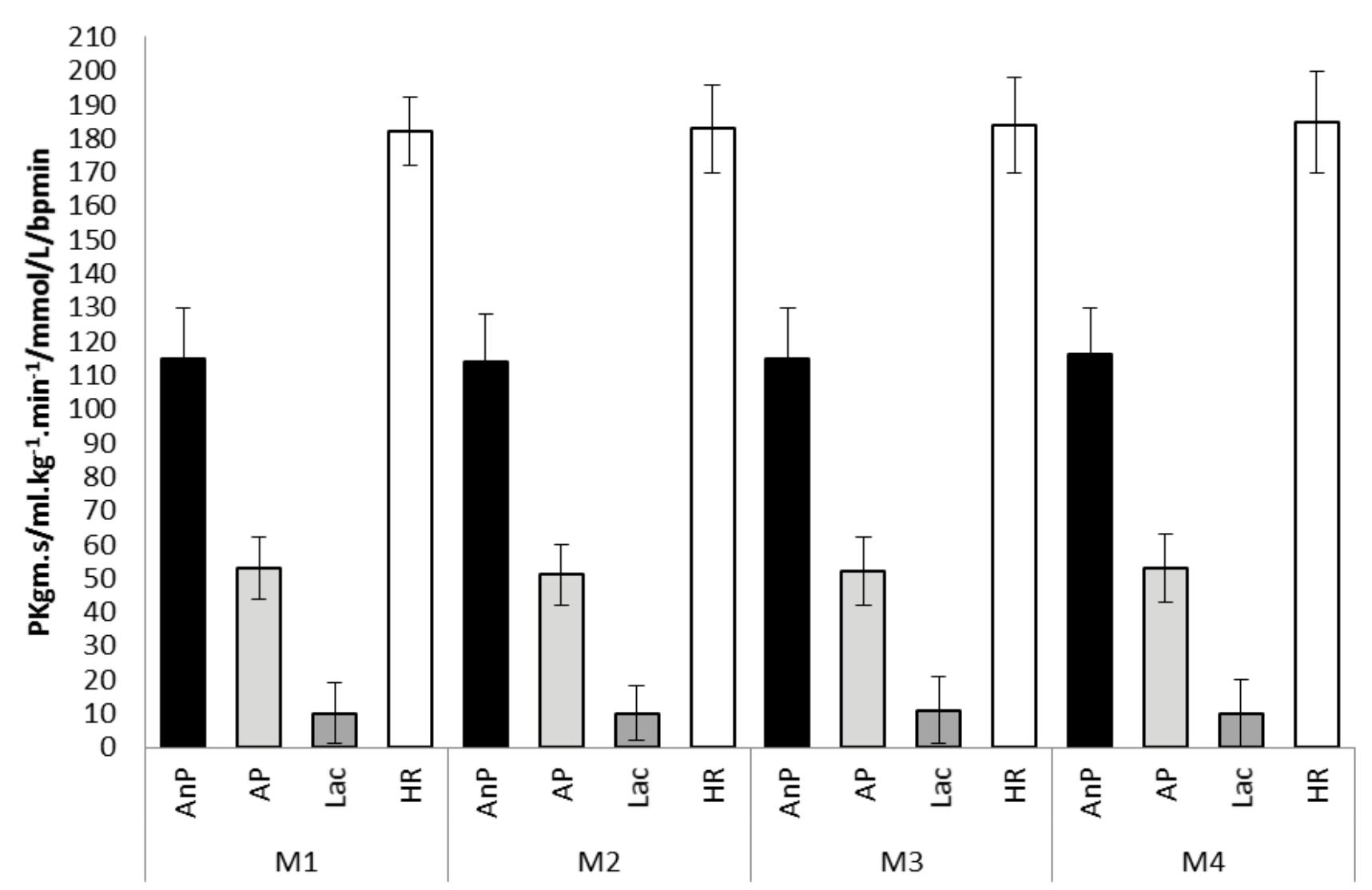

Figure 2: Results of Anaerobic Power (AnP) and Aerobic Power (AP) tests (vertical jump and Yo-Yo test respectively), lactate (Lac) and HR (CF) post-tests in four moments. Tests preceded by traditional warm-up (TW, M1 and M3), or proprioceptive neuromuscular facilitation stretching (PNF, M2 and M4), with an interval of 1 week between them.

$\mathrm{N}=10$; ANOVA; One-way Analysis of Variance, followed by Tukey-Kramer post-test; there was no significant difference for any comparison $(p>0.05)$. $Y$-axis units are respective to the order of the variables.

a study of performance of a specific professional ballet exercise, it was concluded that when warming-up was performed, there was greater participation of the aerobic and less by the anaerobic system in the energy production of this exercise [24]. This justified the economy of movement in ballet, differently of ours observations, in which general sporting movements were used.

\section{Stretching}

Apparently activities that need a higher degree of flexibility, such as fighting, dances, gymnastics benefit from previously performing stretching exercises, however, activities such as jogging or cycling show no evidences of this improvement [16], similar to the findings observed in the present study. In the following paragraphs the authors present studies that investigated the performance of strength/power, aerobic exercises, or DOMS that use different stretching which strategy.

\section{Performance of strength}

Impaired: A difference in isometric contraction of the posterior muscle of the thigh at different angles $\left(90^{\circ}, 70^{\circ}, 50^{\circ}, 30^{\circ}\right)$ was observed, after PNF and static stretching. PNF stretching led to greater loss of strength when compared with static stretching, with the latter being recommended [15]. Trained young soccer players should avoid passive stretching and PNF because of a negative consequence later to vertical jump performance [25]. We observed no loss of power with PNF, which has a direct relationship with the production of strength. The total time of stretching may partly explain these differences. In the study of Balle, et al. [15] they used $60 \mathrm{~s}$ repeated 6 times with an interval of $1 \mathrm{~s}$ with sub maximum isometric contraction of $10 \mathrm{~s}$, while we used a total of $30 \mathrm{~s}$, being $10 \mathrm{~s}$ at $60 \%$ of maximum isometric contraction. Furthermore, a review of 43 articles concluded that passive stretching reduced strength, but the ballistic type did not [17].

Neutral: We expected that stretching would reduce the tension in the posterior muscles of the thigh, and would increase the elastic potential of the muscles in the AnP test, as was observed by passive stretching performed by football players [9]. However, we did not observe this, which was reinforced by the finding of 49 active adult individuals who presented no difference in torque and strength of extension of the knees, compared with pre-exercise activity between not stretching, passive stretching and active stretching [26].

Improved: Variations among the possible types of stretching may differentiate their effects. In a study with 100 men, they compared three types of stretching before the exercise of countermovement jump test, with 5 minutes being jogging for the three groups, fol- 
lowed by: a) Ballistic stretching of $5 \mathrm{~s}$ for each exercise; b) PNF + ballistic stretching (same performance as the previous one); and c) PNF plus $30 \mathrm{~s}$ of static stretching. The researchers observed that ballistic stretching alone produced better effects on the performance of counter movement jump test [27], confirming that PNF did not potentiate performance. As a possible justification, the dynamics of this exercise also allow the increase in body temperature as it is closer to the dynamics of the movement used in the AnP test.

Moreover, the types of stretching presented other conflicting results. Sekir, et al. [28] observed that although passive stretching did not prejudice the ratio of strength of the hamstrings/quadriceps, it reduced the strength in both groups measured, and in the isokinetic evaluation at $60 \% \mathrm{~s}$ and $180 \% \mathrm{~s}$ pre- and post-stretching sessions. However, the cited authors concluded that in dynamic activities such as collective games, passive stretching did not harm the performance, differently from Akbulut and Agopyan [13], in which PNF stretching improved the kicking velocity performance of football players. In trained young soccer players the active and ballistic stretching and can be used before vertical jump and sprint activities with the aim of increasing flexibility $[25,29]$. The differentiated training and effects chronic adaptation to training and acute effect of each protocol must be considered. Therefore, we did not use athletes, but active individuals, to minimize interferences of the sports peculiarities. In any event, the results observed were similar to those of ballistic stretching in other studies [13].

\section{Aerobics}

Few studies were found using stretching pre-aerobic activity. Similarly to the present study, Belkhiria-Turki, et al. [30] observed no difference with combinations of passive and active stretching and aerobic exercise in the performance of the stair climbing test.

\section{Delayed onset of muscle soreness}

Stretching exercises are also linked to prevention, reduction in lesions and DOMS after exercise. In health individuals the dynamic contract-relax and static stretching do not provide proven evidence in relieving calf muscle DOMS symptoms [31]. Hebert, et al. [18] concluded that they are not effective for this purpose. In our study, there was no report of DOMS, and it is important to consider that this is more associated with high intensity eccentric exercise than power activities of short duration and aerobic activities of medium duration, as performed in our study. Moreover, the volunteers of the present study were active and DOMS is natural in non-routine activities [18].

\section{Duration of exercise}

Variables such as total time and number of bouts may influence the results of stretching exercises. Stud- ies have investigated different bouts and times of static stretching $(20,30,40,60 \mathrm{~s})$ in a single bout (Group 1 ) or divided into bouts of $10 \mathrm{~s}$ each (Group 2), and found no difference between the groups, observing that the stretching exercises of up to $30 \mathrm{~s}$ improved the performance of velocity when compared with the control without stretching. Stretching for longer than $30 \mathrm{~s}$ did not improve performance [32]. Thus, our protocol was similar to that found in the cited study, because two series of 10 s of static stretching were performed, interspersed with 10s of isometric exercises, however they did not interfere in the power of the test applied. Although the tests performed were different, speed and power are related with regard to performance.

\section{Warming-up $x$ stretching}

Three studies compared different combinations of stretching and specific pre-physical exercise warming-up exercises. They compared ballistic stretching, intermittent isometric exercises, specific warming up in resistance exercises, running and jumping, and found distinct results:

a. One study, with 21 school football players of 20.14 \pm 1.65 compared rest (control), ballistic stretching, prolonged intermittent isometric exercise of low intensity and the same exercise in addition to $30 \%$ of body weight, before they performed the counter movement jump test (AnP), $15 \mathrm{~m}$ running test and agility test (modified agility T-test). Better general performance was observed when pre-test stretching was done, however, without difference in agility, and it was concluded that intermittent isometric exercise of low intensity could be used as an alternative to dynamic stretching before power activities [19] Performing TW and PNF in our study did not interfere in the tests and physiological markers. Not having a control group without exercise limited our conclusion, but the favorable fact is that TW had no effect on increasing articular and muscular amplitude, and PNF did not increase body temperature, suggesting that neither of these conditions changed the performance in the tests. It was also limiting to directly compare PNF with ballistic stretching due to the dynamic nature of the latter, and of TW with intermittent isometric contraction, due to stimulation of contraction differing between them. Moreover, there was no randomization in the order of the tests among the volunteers in the study of Pojskić, et al. [23] which was a limitation. As a solution, we performed crossover in 4 time intervals, avoiding superimposition of learning on the results obtained.

b. With regard to the performance of exerting force against resistance, researchers investigated the effect of ballistic stretching, passive stretching and specific pre-training warming-up for resistance exercises in nine men, performing a maximum number of repetitions in three series of $12 \mathrm{RM}$ for the leg 
press, knee extension, knee flexion and plantar flexion exercises. Six sessions were performed with an interval of $48 \mathrm{~h}$ between them. Although the knee extension exercises obtained the highest number of repetitions, in the general score of the exercises, the specific warming up was significantly better than the stretching exercises. The cited authors concluded by suggesting that stretching exercises must be avoided before strength exercises [10]. In our study, the power exercises did not differ between TW and PNF, and this performance was not harmed.

c. Young and Behm [33], compared the effects of running (4 min), static stretching of knee extensor muscles and performing jumps before an AnP test. The authors showed that static stretching as warm-up produced the lowest values, while the combination of running or running and static stretching and the performance of jumps produced higher values for the production of explosive strength. The cited study obtained results differing from those we observed, because warming-up per se improved the performance, and was potentiated by the jumping exercises.

\section{Warming-up and ambient temperature}

The hotter the environment, the faster physiological warm-up is achieved. The researchers investigated 10 football players submitted to a test of 10 high intensity intermittent series of $6 \mathrm{~s}$ with $34 \mathrm{~s}$ of rest, and previously submitted to warming up by 10 minutes of running; or submersion in water at $40^{\circ} \mathrm{C}$. They observed that both presented better performance than the control group without warming-up, but without differences between them [4]. In our study, as the volunteers were submitted to tests in a warm environment, the advantage that there would be of elevating body temperature in an exercise with aerobic warming-up could have equalized TW and PNF neutralized possible differences. By one side this limited comparison, but on the other hand, this was one of the few studies conducted under these conditions, suggesting that in hot environments at least, warming-up is not necessary.

However, when the individual is submitted to a sub maximum exercise in a relatively hot environment, this diminishes his capacity to perform certain work. Apart from the possible effects on the circulatory system, blood volume and rate of transpiration, exercise performed at high temperatures may also influence cellular metabolism [3,34]. Studies have observed the reduction in the performance of football players submitted to high temperature $\left(34{ }^{\circ} \mathrm{C}\right)$ and humidity [5]. In spite of the ambient temperature in our tests having been higher $\left(35-37^{\circ} \mathrm{C}\right)$, the TW does not seem to have been superimposed.

\section{Gender}

In a comparative study with 31 female schoolchildren aged $17.3 \pm 0.5$ years the protocols of a) $3 \mathrm{~min}$ of jogging plus 7 min of static stretching; b) 3 min of jogging plus $7 \mathrm{~min}$ of dynamic stretching; and c) $3 \mathrm{~min}$ of jogging plus $7 \mathrm{~min}$ of rest, the researchers observed that dynamic stretching favored balance and agility when compared with the other treatments [11]. However, Chatzopoulos, et al. [12] repeated the procedures of the previously cited study, with 27 male schoolchildren aged $17.3 \pm 0.2$ years, establishing $5 \mathrm{~min}$ as the time of stretching and resting, and observed no improvement in the same tests as those applied to the group of girls. However, PNF has different dynamics of performance from the dynamics of stretching or static only, limiting this direct comparison. Contrary to that observed for girls, in our volunteers we observed no difference in favor of stretching, which were different from another study [35]. Avoiding differentiation due to gender, we opted to study young men, compared with the greater portion of studies. Additional warm-up in the cited studies may have influenced in favor of performance, however, all received the same treatment. To avoid this superimposition, we performed interventions in distinct time intervals in crossover format. Nevertheless, all the volunteers performed the same time of jogging, and this difference could be attributed to the type of stretching used [11].

\section{Limitations of the present study and researches with pre-exercise activities}

There are an infinite number of possible variables in pre-exercise activities that prevent them from being joined in a single category. Results may be conflicting when these variables are altered among the studies. Comparison between studies with warm-up only, or warm-up associated with stretching present different results and may depend on many variables, such as; duration of exercise, ambient temperature [4]; gender $[11,12,36]$; order of pre-exercise activities and physical tests [15]. In the present study the temperature may have minimized the effect of warm-up and to resolve the problem, the order of the exercises was performed in the cross-over system in four time intervals, alternating the pre-exercise protocols and using active, but non-athlete individuals thereby reducing the interference of the particularities of each modality.

\section{Conclusions}

Traditional warm-up with increase in body temperature and without increase in muscle amplitude did not differ from PNF stretching that does not increase the body temperature, but increases the articular amplitude in both anaerobic and aerobic physical performance. The data suggested that warm-up or PNF at temperatures of over $30^{\circ} \mathrm{C}$ did not change the performance of anaerobic an aerobic power in active young men.

\section{Contribuições dos Autores}

Each author contributed individually and significantly to the development of the manuscript. 
Carlos Alexandre Fett: Elaboration of the entire research project, study design, intellectual concept, contribution to the conception, writing, selection of participants and data collection, review and data analysis, editing, statistical analysis, discussion of results and execution of the review.

Karla Régia Ferreira Viana Figueiredo: Writing, selection of participants and data collection, review, discussion of results and execution of the review.

Almir de França Ferraz: Writing, selection of participants and data collection, review and analysis of data, editing, statistical analysis, discussion of results and execution of the review.

Fabíula Lebbeck: Review and correction analysis.

Camila Fernanda Cunha Brandão: Editing, revision and tabulation of data.

RLR: Critical review, text analysis and writing.

Waléria Christiane Rezende Fett: Guidance on project design and study design, selection of participants and data collection, discussion of results, intellectual concept, contribution to the design and editing and review. All authors reviewed and approved the final version of the manuscript.

\section{References}

1. Hartmann $H$, Wirth $K$, Keiner M, Mickel C, Sander A, et al. (2015) Short-term Periodization Models: Effects on Strength and Speed-strength Performance. Sport Med 45: 1373-1386.

2. McGowan CJ, Pyne DB, Thompson KG, Rattray B (2015) Warm-Up Strategies for Sport and Exercise: Mechanisms and Applications. Sport Med 45: 1523-1546.

3. Racinais S, Cocking S, Périard JD (2017) Sports and environmental temperature: From warming-up to heating-up. Temperature 4: 227-257.

4. Brown PI, Hughes MG, Tong RJ (2008) The Effect of WarmUp on High-Intensity, Intermittent Running Using Nonmotorized Treadmill Ergometry. J Strength Cond Res 22: 801808.

5. Özgünen KT, Kurdak SS, Maughan RJ, Zeren Ç, Korkmaz S, et al. (2010) Effect of hot environmental conditions on physical activity patterns and temperature response of football players. Scand J Med Sci Sport 20: 140-147.

6. Ehlert A, Wilson PB (2019) A Systematic Review of Golf Warm-ups: Behaviors, Injury, and Performance. J Strength Cond Res.

7. blazevich AJ, Gill ND, Kvorning T, Kay AD, Goh AG, et al. (2018) No Effect of Muscle Stretching within a Full, Dynamic Warm-up on Athletic Performance. Med Sci Sport Exerc 50: 1258-1266.

8. Opplert J, Babault N (2018) Acute Effects of Dynamic Stretching on Muscle Flexibility and Performance: An Analysis of the Current Literature. Sport Med 48: 299-325.

9. Marshall PWM, Lovell R, Siegler JC (2016) Changes in passive tension of the hamstring muscles during a simulated soccer match. Int J Sports Physiol Perform 11: 594-601.

10. Sá MA, Neto GR, Costa PB, Gomes TM, Bentes CM, et al.
(2015) Acute effects of different stretching techniques on the number of repetitions in a single lower body resistance training session. J Hum Kinet 45: 177-185.

11. Chatzopoulos D, Galazoulas C, Patikas D, Kotzamanidis C (2014) Acute effects of static and dynamic stretching on balance, agility, reaction time and movement time. J Sports Sci Med 13: 403-409.

12. Chatzopoulos DE, Yiannakos A, Kotzamanidou M, Bassa E (2015) Warm-up protocols for high school students. Percept Mot Skills 121: 1-13.

13. Akbulut T, Agopyan A (2015) Effects of an Eight-Week Proprioceptive Neuromuscular Facilitation Stretching Program on Kicking Speed and Range of Motion in Young Male Soccer Players. J Strength Cond Res 29: 3412-3423.

14. Reis E da FS, Pereira GB, de Sousa NMF, Tibana RA, Silva MF, et al. (2013) Acute effects of proprioceptive neuromuscular facilitation and static stretching on maximal voluntary contraction and muscle electromyographical activity in indoor soccer players. Clin Physiol Funct Imaging 33: 418422.

15. Balle SS, Magnusson SP, Mchugh MP (2015) Effects of contract-relax vs static stretching on stretch-induced strength loss and length-tension relationship. Scand J Med Sci Sport 25: 764-769.

16. Gremion $G$ (2005) Is stretching for sports performance still useful? A review of the literature. Rev Med Suisse 1: 18301834.

17. Kallerud H, Gleeson N (2013) Effects of Stretching on Performances Involving Stretch-Shortening Cycles. Sport Med 43: 733-750.

18. Pastre Carlos Marcelo, Bastos Fábio do Nascimento, Netto Júnior Jayme, Vanderlei Luiz Carlos Marques, Hoshi Rosangela Akemi (2009) Métodos de recuperação pós-exercício: uma revisão sistemática. Rev Bras Med Esporte. 15: $138-144$.

19. Silveira LC, Fett CA (2011) Comparison of four methods in recovery delayed onset muscle soreness. Federal University of Mato Grosso.

20. http://sonda.ccst.inpe.br/estacoes/cuiaba_clima.html

21. Vannucchi H, Marchini J, JEdos S, Oliveira JD de (1984) Avaliação antropométrica e bioquímica do Estado nutricional. Rev da Fac Med Ribeirão Preto 17: 17-28.

22. Kwak DH, Ryu YU (2015) Applying proprioceptive neuromuscular facilitation stretching: Optimal contraction intensity to attain the maximum increase in range of motion in young males. J Phys Ther Sci 27: 2129-2132.

23. Pojskić H, Pagaduan JC, Babajić F, Užičanin E, Muratović $M$, et al. (2015) Acute effects of prolonged intermittent low-intensity isometric warm-up schemes on jump, sprint, and agility performance in collegiate soccer players. Biol Sport 32: 129-134.

24. Guidetti L, Emerenziani G Pietro, Gallotta MC, Baldari C (2007) Effect of warm up on energy cost and energy sources of a ballet dance exercise. Eur J Appl Physiol 99: 275281.

25. Oliveira LP, Vieira LHP, Aquino R, Manechini JPV, Santiago PRP, et al. (2018) Acute Effects of Active, Ballistic, Passive, and Proprioceptive Neuromuscular Facilitation Stretching on Sprint and Vertical Jump Performance in Trained Young Soccer Players. J Strength Cond Res 32: 2199-2208.

26. Ayala F, De Ste Croix M, Sainz De Baranda $P$, Santonja $F$ (2013) Acute effects of static and dynamic stretching on 
hamstring eccentric isokinetic strength and unilateral hamstring to quadriceps strength ratios. J Sports Sci 31: 831839 .

27. Kirmizigil B, Ozcaldiran B, Colakoglu M (2014) Effects of three different stretching techniques on vertical jumping performance. J Strength Cond Res 28: 1263-1271.

28. Sekir U, Arabaci R, Akova B (2015) Acute effects of static stretching on peak and end-range hamstring-to-quadriceps functional ratios. World J Orthop 6: 719-726.

29. Hammami A, Zois J, Slimani M, Russel M, Bouhlel E (2018) The efficacy and characteristics of warm-up and re-warm-up practices in soccer players: A systematic review. J Sports Med Phys Fitness 58: 135-149.

30. Belkhiria-Turki L, Chaouachi A, Turki O, Hammami R, Chtara M, et al. (2014) Greater volumes of static and dynamic stretching within a warm-up do not impair star excursion balance performance. J Sports Med Phys Fitness 54: 279-288.

31. Xie Y, Feng B, Chen K, Andersen LL, Page P, et al. (2018) The Efficacy of Dynamic Contract-Relax Stretching on De-
layed-Onset Muscle Soreness Among Healthy Individuals. Clin J Sport Med 28: 28-36.

32. Avloniti A, Chatzinikolaou A, Fatouros IG, Protopapa M, Athanailidis I, et al. (2016) The effects of static stretching on speed and agility: One or multiple repetition protocols? Eur J Sport Sci 16: 402-408.

33. Young W, Elliott S, Young W, Elliott S (2013) Acute effects of static stretching, proprioceptive voluntary contractions on explosive force production and jumping performance. 3741.

34. Wagner AL, Keusch F, Yan T, Clarke PJ (2019) The impact of weather on summer and winter exercise behaviors. J Sport Health Sci 8: 39-45.

35. Zakas A, Doganis G, Zakas N VA (2006) Acute effects of active warm-up and stretching on the flexibility of elderly women. J Sports Med Phys Fitness 4: 617-622.

36. Fett CA, Christiane W, Fett R, Marchini JS (2009) Exercicio Resistido vs Jogging em Fatores de Risco Metabólicos de Mulheres com Sobrepeso/Obesas. Arq Bras Cardiol 93: 519-525. 